Bull. Korean Math. Soc. 50 (2013), No. 2, pp. 507-524

http://dx.doi.org/10.4134/BKMS.2013.50.2.507

\title{
n-ARY HYPERGROUPS ASSOCIATED WITH $n$-ARY RELATIONS
}

\author{
Seid Mohammad Anvariyeh and Somayyeh Momeni
}

\begin{abstract}
The notion of $n$-ary algebraic hyperstructures is a generalization of ordinary algebraic hyperstructures. In this paper, we associate an $n$-ary hypergroupoid $(H, f)$ with an $(n+1)$-ary relation $\rho_{n+1}$ defined on a non-empty set $H$. Then, we obtain some basic results in this respect. In particular, we investigate when it is an $n$-ary $H_{v}$-group, an $n$-ary hypergroup or a join $n$-ary space.
\end{abstract}

\section{Introduction and basic definitions}

Algebraic hyperstructures represent a natural extension of classical algebraic structures and they were introduced by Marty [14]. The connections between hyperstructures and binary relations have been analyzed by many researchers, such as Corsini [1], Corsini and Leoreanu [2], De Salvo and Lo Faro [7, 8], Leoreanu and Leoreanu [13], Rosenberg [16], Rasouli and Davvaz [15], Spartalis [17], Spartalis and Mamaloukas [18] and so on. $n$-ary generalizations of algebraic structures is the most natural way for further development and deeper understanding of their fundamental properties. In [6], Davvaz and Vougiouklis introduced the concept of $n$-ary hypergroups as a generalization of hypergroups in the sense of Marty. Also, we can consider $n$-ary hypergroups as a nice generalization of $n$-ary groups. In [11], Leoreanu-Fotea and Davvaz introduced and studied the notion of a partial $n$-ary hypergroupoid associated with a binary relation. Some important results concerning Rosenberg partial hypergroupoids, induced by relations, are generalized to the case of $n$-ary hypergroupoids. Then, $n$-ary hypergroups associated with union, intersection, products of relations and also mutually associative $n$-ary hypergroupoids are analyzed. Also, in [5], they investigated binary relations on ternary semihypergroups and studied some basic properties of binary relations on them. Davvaz and et al. in [4] considered a class of algebraic hypersystems which represent a generalization of semigroups, semihypergroups and $n$-ary semigroups. In

Received October 26, 2011

2010 Mathematics Subject Classification. 20N20.

Key words and phrases. hypergroup, binary relation, $n$-ary hypergroup, $n$-ary $H_{v}$-group, join $n$-ary space. 
[12], Leoreanu-Fotea and Davvaz studied the rough sets within the context of the commutative $n$-ary hypergroups. In [3], Cristea and Stefanescu extended some results on the hypergroups connected with binary relations to the case of $n$-ary relations. In particular, they established some connections between hypergroupoids associated with $n$-ary relations and hypergroupoids associated with binary or ternary relations.

Let $H$ be a non-empty set and $f$ a mapping $f: H^{n} \longrightarrow \wp^{*}(H)$, where $\wp^{*}(H)$ is the set of all non-empty subsets of $H$. Then, $f$ is called an $n$-ary hyperoperation on $H$. We denoted by $H^{n}$ the Cartesian product $H \times \cdots \times H$, where $H$ appears $n$ times and an element of $H^{n}$ will be denoted by $\left(x_{1}, \ldots, x_{n}\right)$, such that $x_{i} \in H$ for any $i$ with $1 \leq i \leq n$. In general, a mapping $f$ : $H^{n} \longrightarrow \wp^{*}(H)$ is called an $n$-ary hyperoperation and $n$ is called the arity of hyperoperation. Let $f$ be an $n$-ary hyperoperation on $H$ and $A_{1}, \ldots, A_{n}$ be non-empty subsets of $H$. We define $f\left(A_{1}, \ldots, A_{n}\right)=\cup\left\{f\left(x_{1}, \ldots, x_{n}\right) \mid x_{i} \in\right.$ $\left.A_{i}, i=1, \ldots, n\right\}$. We shall use the following abbreviated notation: the sequence $x_{i}, x_{i+1}, \ldots, x_{j}$ will be denoted by $x_{i}^{j}$. Also, for every $a \in H$, we write $f(\underbrace{a, \ldots, a}_{n})=f(\stackrel{(n)}{a})$ and for $j<i, x_{i}^{j}$ is the empty set. In this convention $f\left(x_{1}, \ldots, x_{i}, y_{i+1}, \ldots, y_{j}, x_{j+1}, \ldots, x_{n}\right)$ will be written $f\left(x_{1}^{i}, y_{i+1}^{j}, x_{j+1}^{n}\right)$. A non-empty set $H$ with an $n$-ary hyperoperation $f: H^{n} \longrightarrow \wp^{*}(H)$ will be called an $n$-ary hypergroupoid and will be denote by $(H, f)$. An $n$-ary hypergroupoid $(H, f)$ is commutative if for all $\sigma \in \mathbb{S}_{n}$ and for every $a_{1}^{n} \in H$, we have $f\left(a_{1}^{n}\right)=f\left(a_{\sigma(1)}^{\sigma(n)}\right)$. An $n$-ary hypergroupoid $(H, f)$ is called an $n$-ary semihypergroup if for any $i, j \in\{1,2, \ldots, n\}$ and $a_{1}^{2 n-1} \in H$, we have

$$
f\left(a_{1}^{i-1}, f\left(a_{i}^{n+i-1}\right), a_{n+i}^{2 n-1}\right)=f\left(a_{1}^{j-1}, f\left(a_{j}^{n+j-1}\right), a_{n+j}^{2 n-1}\right) \quad \text { (associative law). }
$$

An $n$-ary hypergroupoid $(H, f)$, in which the equation $b \in f\left(a_{1}^{i-1}, x_{i}, a_{i+1}^{n}\right)$ has a solution $x_{i} \in H$ for every $a_{1}, \ldots, a_{i-1}, a_{i+1}, \ldots, a_{n}, b \in H$ and $1 \leq i \leq n$, is called a quasi $n$-ary hypergroup. A quasi $n$-ary hypergroup $(H, f)$ with the associative law is called an $n$-ary hypergroup. An $n$-ary hypergroupoid $(H, f)$ is called an $n$-ary $H_{v}$-semigroup if the following week associative axiom holds:

$$
\bigcap_{i=1}^{2 n-1} f\left(x_{1}^{i-1}, f\left(x_{i}^{n+i-1}\right), x_{n+i}^{2 n-1}\right) \neq \emptyset
$$

for any $x_{1}, x_{2}, \ldots, x_{2 n-1} \in H$. An $n$-ary $H_{v}$-semigroup $(H, f)$ in which is a quasi $n$-ary hypergroup is called an $n$-ary $H_{v}$-group. Note that the notion of $n$-ary $H_{v}$-group is a generalization of $H_{v}$-group $[20,21]$.

\section{2. $n$-ary relations}

In this section, we present some basic results about the $n$-ary relations. Suppose that $H$ is a non-empty set and $\rho \subseteq H^{n}$ is an $n$-ary relation on $H$. We recall the following definition from [3]. 
Definition 2.1. The relation $\rho$ is said to be

(1) reflexive, if for any $x \in H$, the $n$-tuple $(x, \ldots, x) \in \rho$;

(2) $n$-transitive if it has the following property: if $\left(x_{1}, \ldots x_{n}\right) \in \rho,\left(y_{1}, \ldots y_{n}\right)$ $\in \rho$ hold and if there exist natural numbers $i_{0}>j_{0}$ such that $1<i_{0} \leq n$, $1 \leq j_{0}<n, x_{i_{0}}=y_{j_{0}}$, then the $n$-tuple $\left(x_{i_{1}}, \ldots, x_{i_{k}}, y_{j_{k+1}}, \ldots, y_{j_{n}}\right) \in \rho$, for any natural number $1 \leq k<n$ and $i_{1}, \ldots, i_{k}, j_{k+1}, \ldots, j_{n}$ such that $1 \leq i_{1}<$ $\ldots<i_{k}<i_{0}, j_{0}<j_{k+1}<\ldots<j_{n} \leq n$

(3) symmetric if $\left(x_{1}, x_{2}, \ldots, x_{n}\right) \in \rho$ implies $\left(x_{n}, x_{n-1}, \ldots, x_{1}\right) \in \rho$;

(4) strongly symmetric if $\left(x_{1}, x_{2}, \ldots, x_{n}\right) \in \rho$ implies $\left(x_{\sigma(1)}, \ldots, x_{\sigma(n)}\right) \in \rho$ for any permutation $\sigma$ of the set $\{1, \ldots, n\}$;

(5) $n$-ary preordering on $H$ if it is reflexive and $n$-transitive;

(6) $n$-equivalence on $H$ if it is reflexive, strongly symmetric and $n$-transitive.

Example 1. Let $H=\mathbb{C}$ (complex numbers) and $\left(x_{1}, \ldots, x_{n}\right) \in \rho$ when $\left|x_{1}\right|=$ $\left|x_{2}\right|=\cdots=\left|x_{n}\right|$. Then, $\rho$ is reflexive, strongly symmetric and $n$-transitive.

Example 2. Let $H=\mathbb{N}$ (natural numbers) and $\left(x_{1}, \ldots, x_{n}\right) \in \rho$ when $x_{1}<$ $x_{2}<\cdots<x_{n}$. It is easily to see that $\rho$ is $n$-transitive but it is not reflexive and strongly symmetric.

Definition 2.2. Let $\rho$ be an $n$-ary relation on a set $H$. For any $x \in H$ and any $i \in\{1, \ldots, n\}$ and $k \in\{1, \ldots, n-(i+1)\}$, we define:

$$
\begin{aligned}
L_{i}(x)=\{y \in H \mid & \exists u_{1}, \ldots, u_{n-2} \in H:\left(y, u_{1}, \ldots, u_{i-1}, x, u_{i}, \ldots, u_{n-2}\right) \in \rho \\
& \left.\vee\left(u_{1}, \ldots, u_{k}, y, u_{k+1}, \ldots, u_{k+i-1}, x, u_{k+i}, \ldots, u_{n-2}\right) \in \rho\right\},
\end{aligned}
$$

and

$$
\begin{aligned}
R_{i}(x)=\{y \in H \mid & \exists u_{1}, \ldots, u_{n-2} \in H:\left(x, u_{1}, \ldots, u_{i-1}, y, u_{i}, \ldots, u_{n-2}\right) \in \rho \\
& \left.\vee\left(u_{1}, \ldots, u_{k}, x, u_{k+1}, \ldots, u_{k+i-1}, y, u_{k+i}, \ldots, u_{n-2}\right) \in \rho\right\} .
\end{aligned}
$$

Example 3. In Example 1, for any $x \in H$ and $i \in\{2, \ldots, n-1\}$, we have

$$
L_{i}(x)=R_{i}(x)=\{z \in \mathbb{C}|| z|=| x \mid\} .
$$

Example 4. In Example 2, for any $x \in H$ and $i \in\{1, \ldots, n\}$, we have

$$
\begin{aligned}
& L_{i}(x)=\{y \in \mathbb{N} \mid y<x+i\}, \\
& R_{i}(x)=\{y \in \mathbb{N} \mid y>x+i\} .
\end{aligned}
$$

Remark 1 . Let $\rho$ be an $n$-ary relation on a set $H$. Then, it is obvious that

(1) $y \in L_{i}(x)$ if and only if $x \in R_{i}(y)$ for any $(x, y) \in H^{2}$ and any $i$ $\in\{1, \ldots, n\}$.

(2) $L_{i}(H)=\bigcup_{x \in H} L_{i}(x) \neq H$ if and only if there exists $y \in H$ such that $R_{i}(y)=\emptyset$,

(3) $R_{i}(H)=\bigcup_{x \in H} R_{i}(x) \neq H$ if and only if there exists $y \in H$ such that $L_{i}(y)=\emptyset$,

(4) $x \notin L_{i}(H)$ if and only if $R_{i}(x)=\emptyset$,

(5) $x \notin R_{i}(H)$ if and only if $L_{i}(x)=\emptyset$. 
Indeed, $\bigcup_{x \in H} L_{i}(x) \neq H$ if there exists $y \in H$ such that $y \notin \bigcup_{x \in H} L_{i}(x)$, which is equivalent to the fact there exists $y \in H$ such that $y \notin L_{i}(x)$ for any $x \in H$, equivalent to the fact that there exists $y \in H$ such that $R_{i}(y)=\emptyset$.

Definition 2.3. Let $\rho$ be an $n$-ary relation on the non-empty set $H$. Set $m=\left[\frac{n+1}{2}\right]$. We define on $H$ the following $n$-ary hyperoperation:

$$
f_{\rho}\left(x_{1}, \ldots, x_{n}\right)=\bigcup_{i=1}^{m} L_{i}\left(x_{i}\right) \cup \bigcup_{i=1}^{m} R_{i}\left(x_{n-i+1}\right) .
$$

We notice that if $\left(H, f_{\rho}\right)$ is an $n$-ary hypergroupoid, then $L_{i}(x) \neq \emptyset$ or $R_{i}(x) \neq \emptyset$ for some $x \in H$ and $i \in\{1, \ldots, m\}$.

Theorem 2.4. Let $\rho$ be an $n$-ary relation on the non-empty set $H$. The $n$-ary hypergroupoid $\left(H, f_{\rho}\right)$ is a quasi $n$-ary hypergroup if and only if for any $x \in H$ and any $1 \leq i \leq m, L_{i}(x) \neq \emptyset$ and $R_{i}(x) \neq \emptyset$.

Proof. Let for any $x \in H$ and for any $1 \leq i \leq m, L_{i}(x) \neq \emptyset$ and $R_{i}(x) \neq \emptyset$. Then, $L_{i}(H)=H$ and $R_{i}(H)=H$. So, for every $x_{1}, \ldots, x_{n} \in H$, we have

$$
\begin{aligned}
& f_{\rho}\left(H, x_{2}, \ldots, x_{n}\right) \\
= & L_{1}(H) \cup L_{2}\left(x_{2}\right) \cup \cdots \cup L_{m}\left(x_{m}\right) \cup R_{1}\left(x_{n}\right) \cup \cdots \cup R_{m}\left(x_{n-m+1}\right)=H, \\
& f_{\rho}\left(x_{1}, H, \ldots, x_{n}\right) \\
= & L_{1}\left(x_{1}\right) \cup L_{2}(H) \cup \cdots \cup L_{m}\left(x_{m}\right) \cup R_{1}\left(x_{n}\right) \cup \cdots \cup R_{m}\left(x_{n-m+1}\right)=H, \\
& \quad \vdots \\
& f_{\rho}\left(x_{1}, \ldots, H, x_{n}\right) \\
= & L_{1}\left(x_{1}\right) \cup \cdots \cup L_{m}\left(x_{m}\right) \cup R_{1}\left(x_{n}\right) \cup R_{2}(H) \cup \cdots \cup R_{m}\left(x_{n-m+1}\right)=H, \\
& f_{\rho}\left(x_{1}, \ldots, x_{n-1}, H\right) \\
= & L_{1}\left(x_{1}\right) \cup \cdots \cup L_{m}\left(x_{m}\right) \cup R_{1}(H) \cup R_{2}\left(x_{n-1}\right) \cup \cdots . \\
& \cup R_{m-1}\left(x_{n-m+2}\right) \cup R_{m}\left(x_{n-m+1}\right)=H .
\end{aligned}
$$

Thus, $\left(H, f_{\rho}\right)$ is reproductive, so it is a quasi $n$-ary hypergroup.

Conversely, suppose that $\left(H, f_{\rho}\right)$ is a quasi $n$-ary hypergroup and for some $i \in\{1, \ldots, m\}$, there exists $x \in H$ such that $L_{i}(x)=\emptyset$ or $R_{i}(x)=\emptyset$.

If $L_{i}(x)=\emptyset$, then $x \notin R_{i}(H)$. Also, it easy to see that for any $j \in\{1, \ldots, m\}$, $x \notin L_{j}(x)$ (also $x \notin R_{j}(x)$ ). Therefore,

$$
\begin{aligned}
x \notin & L_{1}(x) \cup \cdots \cup L_{m}(x) \cup R_{1}(x) \cup \cdots \cup R_{i}(H) \cup \cdots \cup R_{m}(x) \\
= & f_{\rho}(x, \ldots, H, \ldots, x)=H,
\end{aligned}
$$

where $H$ is in the $i$-place and this contradicts the reproducibility low. If $R_{i}(x)$ $=\emptyset$, the similar argument implies a contradiction.

Example 5. Let $H=\{1,2,3,4\}$ and

$$
\rho=\{(\underbrace{1,1, \ldots, 1}_{n-1}, 2),(3, \underbrace{1,1, \ldots, 1}_{n-2}, 3),(2, \underbrace{3, \ldots, 3}_{n-2}, 1),
$$




$$
(\underbrace{2, \ldots, 2}_{n-1}, 3),(3, \underbrace{4, \ldots, 4}_{n-1}),(\underbrace{4, \ldots, 4}_{n-1}, 1)\} .
$$

Now, for any $x \in H$ and $1 \leq i \leq m$, we have

\begin{tabular}{|c|c|c|c|c|c|c|c|c|c|}
\hline & $L_{1}$ & $L_{2}$ & $L_{3}$ & $\ldots$ & $L_{m}$ & $R_{1}$ & $R_{2}$ & $\ldots$ & $R_{m}$ \\
\hline 1 & $\{1,3,4\}$ & $\{1,3,4\}$ & $\{1,3,4\}$ & $\ldots$ & $\{1,3,4\}$ & $\{1,2,3\}$ & $\{1,2,3\}$ & $\ldots$ & $\{1,2,3\}$ \\
\hline 2 & $\{1,2\}$ & $\{1,2\}$ & $\{1,2\}$ & $\ldots$ & $\{1,2\}$ & $\{2,3\}$ & $\{2,3\}$ & $\ldots$ & $\{2,3\}$ \\
\hline 3 & $\{1,2\}$ & $\{1,2\}$ & $\{1,2\}$ & $\ldots$ & $\{1,2\}$ & $\{1,4\}$ & $\{1,4\}$ & $\ldots$ & $\{1,4\}$ \\
\hline 4 & $\{3,4\}$ & $\{3,4\}$ & $\{3,4\}$ & $\ldots$ & $\{3,4\}$ & $\{1,4\}$ & $\{1,4\}$ & $\ldots$ & $\{1,4\}$ \\
\hline
\end{tabular}

$$
\begin{aligned}
& L_{i}(H)=L_{i}(1) \cup L_{i}(2) \cup L_{i}(3) \cup L_{i}(4)=\{1,2,3,4\} \\
& R_{i}(H)=R_{i}(1) \cup R_{i}(2) \cup R_{i}(3) \cup R_{i}(4)=\{1,2,3,4\} .
\end{aligned}
$$

Also,

$$
\begin{aligned}
& f_{\rho}\left(H, x_{2}, \ldots, x_{n}\right) \\
& =\left(L_{1}(H)=\bigcup_{x \in H} L_{1}(x)\right) \cup L_{2}\left(x_{2}\right) \cup \cdots \cup L_{m}\left(x_{m}\right) \cup R_{1}\left(x_{n}\right) \cup \\
& \cdots \cup R_{m}\left(x_{n-m+1}\right) \\
& =\{1,2,3,4\} \cup L_{2}\left(x_{2}\right) \cup \cdots \cup L_{m}\left(x_{m}\right) \cup R_{1}\left(x_{n}\right) \cup \ldots \cup R_{m}\left(x_{n-m+1}\right)=H \text {, } \\
& f_{\rho}\left(x_{1}, H, \ldots, x_{n}\right) \\
& =L_{1}\left(x_{1}\right) \cup\left(L_{2}(H)=\bigcup_{x \in H} L_{2}(x)\right) \cup \cdots \cup L_{m}\left(x_{m}\right) \cup R_{1}\left(x_{n}\right) \cup \\
& \cdots \cup R_{m}\left(x_{n-m+1}\right) \\
& =L_{1}\left(x_{1}\right) \cup\{1,2,3,4\} \cup \cdots \cup L_{m}\left(x_{m}\right) \cup R_{1}\left(x_{n}\right) \cup \cdots \cup R_{m}\left(x_{n-m+1}\right)=H \text {, } \\
& f_{\rho}\left(x_{1}, \ldots, H, x_{n}\right) \\
& =L_{1}\left(x_{1}\right) \cup \cdots \cup L_{m}\left(x_{m}\right) \cup R_{1}\left(x_{n}\right) \cup\left(R_{2}(H)=\bigcup_{x \in H} R_{2}(x)\right) \cup \\
& \cdots \cup R_{m}\left(x_{n-m+1}\right) \\
& =L_{1}\left(x_{1}\right) \cup \cdots \cup L_{m}\left(x_{m}\right) \cup R_{1}\left(x_{n}\right) \cup\{1,2,3,4\} \cup \cdots \cup R_{m}\left(x_{n-m+1}\right)=H \text {, } \\
& f_{\rho}\left(x_{1}, \ldots, x_{n-1}, H\right) \\
& =L_{1}\left(x_{1}\right) \cup \cdots \cup L_{m}\left(x_{m}\right) \cup\left(R_{1}(H)=\bigcup_{x \in H} R_{1}(x)\right) \cup R_{2}\left(x_{n-1}\right) \cup \\
& \cdots \cup R_{m}\left(x_{n-m+1}\right) \\
& =L_{1}\left(x_{1}\right) \cup \cdots \cup L_{m}\left(x_{m}\right) \cup\{1,2,3,4\} \cup R_{2}\left(x_{n-1}\right) \cup \cdots \cup R_{m}\left(x_{n-m+1}\right)=H \text {. }
\end{aligned}
$$

Therefore, the $n$-ary hypergroupoid $\left(H, f_{\rho}\right)$ is a quasi $n$-ary hypergroup. 
Theorem 2.5. Let $\rho$ be an $n$-ary relation on the non-empty set $H$. The $n$-ary hypergroupoid $\left(H, f_{\rho}\right)$ is an $n$-ary $H_{v}$-group if and only if, for any $x \in H$ and $i \in\{1, \ldots, m\}, L_{i}(x) \neq \emptyset$ and $R_{i}(x) \neq \emptyset$.

Proof. If $\left(H, f_{\rho}\right)$ is an $n$-ary $H_{v}$-group, then it is a quasi $n$-ary hypergroup and by Theorem 2.4, it follows that for any $x \in H$ and $i \in\{1, \ldots, m\}, L_{i}(x) \neq \emptyset$ and $R_{i}(x) \neq \emptyset$.

Conversely, suppose that for any $x \in H$ and $i \in\{1, \ldots, m\}, L_{i}(x) \neq \emptyset$ and $R_{i}(x) \neq \emptyset$. By Theorem 2.4, it follows that $\left(H, f_{\rho}\right)$ is a quasi $n$-ary hypergroup. It remains to prove that the $n$-ary hyperoperation $f_{\rho}$ is weakly associative. For this, we show that, for any $x_{1}^{2 n-1} \in H$,

$$
\bigcap_{i=1}^{2 n-1} f_{\rho}\left(x_{1}^{i-1}, f_{\rho}\left(x_{i}^{n+i-1}\right), x_{n+i}^{2 n-1}\right) \neq \emptyset \text {. }
$$

We have

$\left(i_{1}\right)$

$$
\begin{aligned}
& f_{\rho}\left(f_{\rho}\left(x_{1}, \ldots, x_{n}\right), x_{n+1}, \ldots, x_{2 n-1}\right) \\
= & \left\{L_{1}(u) \cup L_{2}\left(x_{n+1}\right) \cup \cdots \cup L_{m}\left(x_{n+m-1}\right) \cup R_{1}\left(x_{2 n-1}\right) \cup \cdots \cup R_{m}\left(x_{2 n-m}\right) \mid\right. \\
& \left.u \in L_{1}\left(x_{1}\right) \cup \cdots \cup L_{m}\left(x_{m}\right) \cup R_{1}\left(x_{n}\right) \cup \cdots \cup R_{m}\left(x_{n-m+1}\right)\right\} \\
\supseteq & \left\{L_{1}(u) \mid u \in L_{1}\left(x_{1}\right) \cup \cdots \cup L_{m}\left(x_{m}\right) \cup R_{1}\left(x_{n}\right) \cup \cdots \cup R_{m}\left(x_{n-m+1}\right)\right\} \\
\supseteq & \left\{L_{1}(u) \mid u \in R_{1}\left(x_{n}\right)\right\}=\left\{L_{1}(u) \mid x_{n} \in L_{1}(u)\right\} \ni x_{n},
\end{aligned}
$$

$\left(i_{2}\right)$

$$
\begin{aligned}
& f_{\rho}\left(x_{1}, f_{\rho}\left(x_{2}, \ldots, x_{n+1}\right), x_{n+2}, \ldots, x_{2 n-1}\right) \\
= & \left\{L_{1}\left(x_{1}\right) \cup L_{2}(u) \cup \ldots \cup L_{m}\left(x_{n+m-1}\right) \cup R_{1}\left(x_{2 n-1}\right) \cup \cdots \cup R_{m}\left(x_{2 n-m}\right) \mid\right. \\
& \left.u \in L_{1}\left(x_{2}\right) \cup \cdots \cup L_{m}\left(x_{m+1}\right) \cup R_{1}\left(x_{n+1}\right) \cup \cdots \cup R_{m}\left(x_{n-m+2}\right)\right\} \\
\supseteq & \left\{L_{1}(u) \mid u \in L_{1}\left(x_{2}\right) \cup \cdots \cup L_{m}\left(x_{m+1}\right) \cup R_{1}\left(x_{n+1}\right) \cup \cdots \cup R_{m}\left(x_{n-m+2}\right)\right\} \\
\supseteq & \left\{L_{2}(u) \mid u \in R_{2}\left(x_{n}\right)\right\}=\left\{L_{2}(u) \mid x_{n} \in L_{2}(u)\right\} \ni x_{n},
\end{aligned}
$$

$$
\begin{aligned}
& \left(i_{n-1}\right) \\
& \quad f_{\rho}\left(x_{1}, \ldots, x_{n-2}, f_{\rho}\left(x_{n-1}, \ldots, x_{2 n-2}\right), x_{2 n-1}\right) \\
& =\left\{L_{1}\left(x_{1}\right) \cup \cdots \cup L_{m}\left(x_{m}\right) \cup R_{1}\left(x_{2 n-1}\right) \cup R_{2}(u) \cup \cdots \cup R_{m}\left(x_{n-m+1}\right) \mid\right. \\
& \left.\quad u \in L_{1}\left(x_{n-1}\right) \cup \cdots \cup L_{m}\left(x_{n+m-2}\right) \cup R_{1}\left(x_{2 n-2}\right) \cup \cdots \cup R_{m}\left(x_{2 n-m-1}\right)\right\} \\
& \supseteq\left\{R_{2}(u) \mid u \in L_{1}\left(x_{n-1}\right) \cup \cdots \cup L_{m}\left(x_{n+m-2}\right) \cup R_{1}\left(x_{2 n-2}\right) \cup\right. \\
& \left.\quad \cdots \cup R_{m}\left(x_{2 n-m-1}\right)\right\} \\
& \supseteq\left\{R_{2}(u) \mid u \in L_{2}\left(x_{n}\right)\right\}=\left\{R_{2}(u) \mid x_{n} \in R_{2}(u)\right\} \ni x_{n}, \\
& \left(i_{n}\right) \\
& f_{\rho}\left(x_{1}, \ldots, x_{n-1}, f_{\rho}\left(x_{n}, \ldots, x_{2 n-1}\right)\right)
\end{aligned}
$$




$$
\begin{aligned}
= & \left\{L_{1}\left(x_{1}\right) \cup \cdots \cup L_{m}\left(x_{m}\right) \cup R_{1}(u) \cup R_{2}\left(x_{n-1}\right) \cup \cdots \cup R_{m}\left(x_{n-m+1}\right) \mid\right. \\
& \left.u \in L_{1}\left(x_{n}\right) \cup \cdots \cup L_{m}\left(x_{n+m-1}\right) \cup R_{1}\left(x_{2 n-1}\right) \cup \cdots \cup R_{m}\left(x_{2 n-m}\right)\right\} \\
\supseteq & \left\{R_{1}(u) \mid u \in L_{1}\left(x_{n}\right) \cup \cdots \cup L_{m}\left(x_{n+m-1}\right) \cup R_{1}\left(x_{2 n-1}\right) \cup \cdots \cup R_{m}\left(x_{2 n-m}\right)\right\} \\
\supseteq & \left\{R_{1}(u) \mid u \in L_{1}\left(x_{n}\right)\right\}=\left\{R_{1}(u) \mid x_{n} \in R_{1}(u)\right\} \ni x_{n} .
\end{aligned}
$$

It follows that $\left(H, f_{\rho}\right)$ is an $n$-ary $H_{v}$-group.

Example 6. Let $H=\{1,2,3\}$ and $\rho=\{(\underbrace{1, \ldots, 1}_{n-2}, 2,1),(2, \underbrace{3, \ldots, 3}_{n-1}),(\underbrace{2, \ldots, 2}_{n})\}$ be an $n$-ary relation on $H$. Now, we have:

\begin{tabular}{|c|c|c|c|c|c|c|c|c|c|}
\hline & $L_{1}$ & $L_{2}$ & $L_{3}$ & $\ldots$ & $L_{m}$ & $R_{1}$ & $R_{2}$ & $\ldots$ & $R_{m}$ \\
\hline 1 & $\{1,2\}$ & $\{1\}$ & $\{1\}$ & $\ldots$ & $\{1\}$ & $\{1,2\}$ & $\{1,2\}$ & $\ldots$ & $\{1,2\}$ \\
\hline 2 & $\{1,2\}$ & $\{1,2\}$ & $\{1,2\}$ & $\ldots$ & $\{1,2\}$ & $\{1,2,3\}$ & $\{2,3\}$ & $\ldots$ & $\{2,3\}$ \\
\hline 3 & $\{2\}$ & $\{2,3\}$ & $\{2,3\}$ & $\ldots$ & $\{2,3\}$ & $\{3\}$ & $\{3\}$ & $\ldots$ & $\{3\}$ \\
\hline
\end{tabular}

Also,

$$
\begin{aligned}
& f_{\rho}(f_{\rho}(\underbrace{1, \ldots, 1}_{n}), \underbrace{1, \ldots, 1}_{n-1})=f_{\rho}(\{1,2\}, 1, \ldots, 1)=\{1,2\}, \\
& f_{\rho}(\underbrace{1, \ldots, 1}_{n-1}, f_{\rho}(\underbrace{1, \ldots, 1}_{n})=f_{\rho}(\underbrace{1, \ldots, 1}_{n-1},\{1,2\})=\{1,2,3\} .
\end{aligned}
$$

This example shows that for every $x \in H$ and for any $i \in\{1, \ldots, m\}$, $L_{i}(x) \neq \emptyset, R_{i}(x) \neq \emptyset$ and $\left(H, f_{\rho}\right)$ is an $n$-ary $H_{v}$-group but it is not an $n$-ary hypergroup.

Corollary 2.6. Let $\rho$ be an n-ary relation on a set $H$. The $n$-ary hypergroupoid $\left(H, f_{\rho}\right)$ is an $n$-ary $H_{v}$-group if and only if it is a quasi n-ary hypergroup.

Lemma 2.7. Let $\rho$ be an $n$-ary preordering on a set $H$. Then, for any $a, x, u \in$ $H$ and $i \in\{1, \ldots, n-1\}$, such that $a \in L_{i}(u)\left[a \in R_{i}(u)\right]$ and $u \in L_{i}(x)$ $\left[u \in R_{i}(x)\right]$, it follows that $a \in L_{i}(x)\left[a \in R_{i}(x)\right]$.

Proof. Let $a, x, u \in H$ such that $a \in L_{i}(u)$ and $u \in L_{i}(x)$. Then, there exist $a_{1}, \ldots, a_{n-2}, b_{1}, \ldots, b_{n-2} \in H$ such that $\left(a, a_{1}, \ldots, a_{i-1}, u, a_{i}, \ldots, a_{n-2}\right) \in \rho$ or $\left(a_{1}, \ldots, a_{k}, a, a_{k+1}, \ldots, a_{k+i-1}, u, a_{k+i} \ldots, a_{n-2}\right) \in \rho$ for any $k \in\{1, \ldots, n-i-$ 1\}. Also, we have $\left(u, b_{1}, \ldots, b_{i-1}, x, b_{i}, \ldots, b_{n-2}\right) \in \rho$ or $\left(b_{1}, \ldots, b_{h}, u, b_{h+1}, \ldots\right.$, $\left.b_{h+i-1}, x, b_{h+i} \ldots, b_{n-2}\right) \in \rho$ for any $h \in\{1, \ldots, n-i-1\}$. In the all of the situations, by $n$-transitivity, we have $a \in L_{i}(x)$. In the similar way from $a \in R_{i}(u)$ and $u \in R_{i}(x)$ implies $a \in R_{i}(x)$.

Definition $2.8([11])$. Let $\left(H, f_{\rho}\right)$ be a commutative $n$-ary hypergroup. For $a, b_{1}, \ldots, b_{n-1} \in H$, we denote $a / b_{1}^{n-1}=\left\{x \mid a \in f_{\rho}\left(x, b_{1}, \ldots, b_{n-1}\right)\right\}$. We say that the commutative $n$-ary hypergroup $\left(H, f_{\rho}\right)$ is a join $n$-ary space, if for any $a, c, b_{1}, b_{2}, \ldots, b_{n-1}, d_{1}, d_{2}, \ldots, d_{n-1} \in H$, the following implication holds:

$$
a / b_{1}^{n-1} \cap c / d_{1}^{n-1} \neq \emptyset \Rightarrow f_{\rho}\left(a, d_{1}, \ldots, d_{n-1}\right) \cap f_{\rho}\left(b_{1}, \ldots, b_{n-1}, c\right) \neq \emptyset .
$$


Example 7. Let $\rho=\{(x, x, \ldots, x) \mid x \in H\}$ be the diagonal $n$-ary relation on a set $H$. Then, $\left(H, f_{\rho}\right)$ is a join $n$-ary space. In fact, for any $i \in\{1, \ldots, m\}$ and $x \in H$, we obtain $L_{i}(x)=R_{i}(x)=\{x\}$ and thus, for any $x_{1}^{n} \in H$, it follows that $f_{\rho}\left(x_{1}, \ldots, x_{n}\right)=f_{\rho}\left(x_{\sigma(1)}, \ldots, x_{\sigma(n)}\right)=\left\{x_{1}, \ldots, x_{n}\right\}$. Also, for any $x_{1}^{n} \in H$, $f_{\rho}\left(H, x_{2}, \ldots, x_{n}\right)=f_{\rho}\left(x_{1}, H, x_{3}, \ldots, x_{n}\right)=\ldots=f_{\rho}\left(x_{1}, \ldots, x_{n-1}, H\right)=H$. Moreover, for any $x_{1}^{2 n-1} \in H$,

(i $\left.i_{1}\right) \quad f_{\rho}\left(f_{\rho}\left(x_{1}, \ldots, x_{n}\right), x_{n+1}, \ldots, x_{2 n-1}\right)$

$=f_{\rho}\left(\left\{x_{1}, \ldots, x_{n}\right\}, x_{n+1}, \ldots, x_{2 n-1}\right)=\left\{x_{1}, \ldots, x_{n}, x_{n+1}, \ldots, x_{2 n-1}\right\}$,

$\left(i_{2}\right) \quad f_{\rho}\left(x_{1}, f_{\rho}\left(x_{2}, \ldots, x_{n+1}\right), x_{n+2}, \ldots, x_{2 n-1}\right)$

$=f_{\rho}\left(x_{1},\left\{x_{2} \ldots, x_{n+1}\right\}, x_{n+2}, \ldots, x_{2 n-1}\right)=\left\{x_{1}, \ldots, x_{n}, x_{n+1}, \ldots, x_{2 n-1}\right\}$,

$\left(i_{n}\right) \quad f_{\rho}\left(x_{1},, x_{n-1}, f_{\rho}\left(x_{n}, \ldots, x_{2 n-1}\right)\right)$

$=f_{\rho}\left(x_{1}, \ldots, x_{n-1},\left\{x_{n}, \ldots, x_{2 n-1}\right\}\right)=\left\{x_{1}, \ldots, x_{n}, x_{n+1}, \ldots, x_{2 n-1}\right\}$.

So, $\left(H, f_{\rho}\right)$ is a commutative $n$-ary hypergroup. It remains to prove that, for any $a, c, b_{1}, b_{2}, \ldots, b_{n-1}, d_{1}, d_{2}, \ldots, d_{n-1} \in H$,

$$
a / b_{1}^{n-1} \cap c / d_{1}^{n-1} \neq \emptyset \Rightarrow f_{\rho}\left(a, d_{1}, \ldots, d_{n-1}\right) \cap f_{\rho}\left(b_{1}, \ldots, b_{n-1}, c\right) \neq \emptyset .
$$

We obtain that

$$
\begin{aligned}
& a / a, b_{1}, \ldots, b_{n-2} \\
= & \left\{x \in H \mid a \in f_{\rho}\left(x, a, b_{1}, \ldots, b_{n-2}\right)\right\} \\
= & \left\{x \in H \mid a \in L_{1}(x) \cup L_{2}(a) \cup L_{3}\left(b_{1}\right) \cup \cdots \cup L_{m}\left(b_{m-2}\right) \cup R_{1}\left(b_{n-2}\right) \cup\right. \\
& \left.\quad \ldots \cup R_{m}\left(b_{n-m-1}\right)\right\} \\
= & H, \\
& a / b_{1}, a, b_{2}, \ldots, b_{n-2} \\
= & \left\{x \in H \mid a \in f_{\rho}\left(x, b_{1}, a, b_{2}, \ldots, b_{n-2}\right)\right\} \\
= & \left\{x \in H \mid a \in L_{1}(x) \cup L_{2}\left(b_{1}\right) \cup L_{3}(a) \cup L_{4}\left(b_{2}\right) \cup \cdots \cup L_{m}\left(b_{m-2}\right) \cup R_{1}\left(b_{n-2}\right) \cup\right. \\
= & H, \\
& \vdots \\
& a / b_{1}, b_{2}, \ldots, b_{n-2}, a \\
= & \left\{x \in H \mid a \in f_{\rho}\left(x, b_{1}, \ldots, b_{n-2}, a\right)\right\} \\
= & \left\{x \in H \mid a \in L_{1}(x) \cup L_{2}\left(b_{1}\right) \cup \ldots \cup L_{m}\left(b_{m-1}\right) \cup R_{1}(a) \cup \cdots \cup R_{m}\left(b_{n-m}\right)\right\} \\
= & H .
\end{aligned}
$$

If $a \neq b_{1}, \ldots, b_{n-1}$, then $a / b_{1}, \ldots, b_{n-1}=\left\{x \in H \mid a \in f_{\rho}\left(x, b_{1}, \ldots, b_{n-1}\right)\right\}$ $=\left\{x \in H \mid a \in\left\{x, b_{1}, \ldots, b_{n-1}\right\}\right\}=\{a\}$. Let $a, c, b_{1}, b_{2}, \ldots, b_{n-1}, d_{1}, d_{2}, \ldots$, 
$d_{n-1} \in H, a / b_{1}^{n-1} \cap c / d_{1}^{n-1} \neq \emptyset$. If there exist $i, j \in\{1,2, \ldots, n-1\}$ such that $a=b_{i}$ or $c=d_{j}$, then $a \in f_{\rho}\left(a, d_{1}, \ldots, d_{n-1}\right) \cap f_{\rho}\left(b_{1}, \ldots, b_{n-1}, c\right)$ or $a \in f_{\rho}\left(a, d_{1}, \ldots, d_{n-1}\right) \cap f_{\rho}\left(b_{1}, \ldots, b_{n-1}, c\right)$. If $a \neq b_{1}, \ldots, b_{n-1}$ and $c \neq$ $d_{1}, \ldots, d_{n-1}$, then $f_{\rho}\left(a, d_{1}, \ldots, d_{n-1}\right) \cap f_{\rho}\left(b_{1}, \ldots, b_{n-1}, c\right) \neq \emptyset$ if and only if $a=c$ and thus $a \in f_{\rho}\left(a, d_{1}, \ldots, d_{n-1}\right) \cap f_{\rho}\left(b_{1}, \ldots, b_{n-1}, c\right)$. In both cases $f_{\rho}\left(a, d_{1}, \ldots, d_{n-1}\right) \cap f_{\rho}\left(b_{1}, \ldots, b_{n-1}, c\right) \neq \emptyset$. Therefore, $n$-ary hypergroup $\left(H, f_{\rho}\right)$ is a join $n$-ary space.

Theorem 2.9. If $\rho$ is an $n$-ary preordering on a set $H$ such that $L_{i}(x)=R_{j}(x)$ for any $x \in H$ and $1 \leq i, j \leq m$, then $\left(H, f_{\rho}\right)$ is a join $n$-ary space.

Proof. Set $L_{i}(x)=R_{j}(x)=L(x)$. Since $\rho$ is reflexive, it follows by Theorem 2.4 , that $\left(H, f_{\rho}\right)$ is a quasi $n$-ary hypergroup. Moreover, since $L_{i}(x)=R_{j}(x)$ for any $x \in H$ and $1 \leq i, j \leq m$, it follows that

$$
\begin{aligned}
f_{\rho}\left(x_{1}, \ldots, x_{n}\right) & =L_{1}\left(x_{1}\right) \cup \cdots \cup L_{m}\left(x_{m}\right) \cup R_{1}\left(x_{n}\right) \cup \cdots \cup R_{m}\left(x_{n-m+1}\right) \\
& =L\left(x_{1}\right) \cup \cdots \cup L\left(x_{m}\right) \cup L\left(x_{m+1}\right) \cup \cdots \cup L\left(x_{n}\right) \\
& =\bigcup_{i=1}^{n} L\left(x_{i}\right)
\end{aligned}
$$

and this implies that $f_{\rho}\left(x_{1}, \ldots, x_{n}\right)=f_{\rho}\left(x_{\sigma(1)}, \ldots, x_{\sigma(n)}\right)$ for any $x_{1}^{n} \in H$ and for any permutation $\sigma \in\{1, \ldots, n\}$. Therefore, $\left(H, f_{\rho}\right)$ is commutative. Now, we prove that the $n$-ary hyperoperation $f_{\rho}$ is associative, that means for any $i, j \in\{1,2, \ldots, n\}$ and $a_{1}^{2 n-1} \in H$, we have

$$
f_{\rho}\left(a_{1}^{i-1}, f_{\rho}\left(a_{i}^{n+i-1}\right), a_{n+i}^{2 n-1}\right)=f_{\rho}\left(a_{1}^{j-1}, f_{\rho}\left(a_{j}^{n+j-1}\right), a_{n+j}^{2 n-1}\right) .
$$

For any $a \in f_{\rho}\left(f_{\rho}\left(x_{1}, \ldots, x_{n}\right), x_{n+1}, \ldots, x_{2 n-1}\right)$, there exists

$$
\begin{aligned}
u \in & L_{1}\left(x_{1}\right) \cup \cdots \cup L_{m}\left(x_{m}\right) \cup R_{1}\left(x_{n}\right) \cup \cdots \cup R_{m}\left(x_{n-m+1}\right) \\
= & L\left(x_{1}\right) \cup \cdots \cup L\left(x_{m}\right) \cup L\left(x_{n}\right) \cup \cdots \cup L\left(x_{n-m+1}\right),
\end{aligned}
$$

such that $a \in L(u) \cup L\left(x_{n+1}\right) \cup \cdots \cup L\left(x_{n+m-1}\right) \cup L\left(x_{2 n-1}\right) \cup \cdots \cup L\left(x_{2 n-m}\right)$. Moreover,

(*)

$$
\begin{aligned}
& f_{\rho}\left(x_{1}, f_{\rho}\left(x_{2}, \ldots, x_{n+1}\right), x_{n+2}, \ldots, x_{2 n-1}\right) \\
= & \left\{L\left(x_{1}\right) \cup L(v) \cup L\left(x_{n+2}\right) \cup \ldots \cup L\left(x_{2 n-1}\right) \mid v \in L\left(x_{2}\right) \cup \ldots \cup L\left(x_{n+1}\right)\right\} .
\end{aligned}
$$

We distinguish the following cases:

$\left(i_{1}\right)$ If $a \in L(u)$ and $u \in L\left(x_{1}\right)$, by Lemma 2.7, $a \in L\left(x_{1}\right)$. Therefore, we have $a \in f_{\rho}\left(x_{1}, f_{\rho}\left(x_{2}, \ldots, x_{n+1}\right), x_{n+2}, \ldots, x_{2 n-1}\right)$.

$\left(i_{2}\right)$ If $a \in L(u)$ and $u \in L\left(x_{2}\right)$, then by $(*), a \in f_{\rho}\left(x_{1}, f_{\rho}\left(x_{2}, \ldots, x_{n+1}\right)\right.$, $\left.x_{n+2}, \ldots, x_{2 n-1}\right)$.

$\left(i_{n}\right)$ If $a \in L(u)$ and $u \in L\left(x_{n}\right)$, then $a \in f_{\rho}\left(x_{1}, f_{\rho}\left(x_{2}, \ldots, x_{n+1}\right), x_{n+2}, \ldots\right.$, $x_{2 n-1}$ ). 
$\left(i_{n+1}\right)$ If $a \in L_{2}\left(x_{n+1}\right)=L\left(x_{n+1}\right)$, then there exist $b_{1}, \ldots, b_{n-2} \in H$ such that $\left(a, b_{1}, x_{n+1}, b_{2}, \ldots, b_{n-2}\right) \in \rho$ or $\left(b_{1}, \ldots, b_{k}, a, b_{k+1}, x_{n+1}, b_{k+2}, \ldots\right.$, $\left.b_{n-2}\right) \in \rho$ for any $k \in\{1,2, \ldots, n-3\}$. For example, if $\left(a, b_{1}, x_{n+1}, b_{2}\right.$, $\left.\ldots, b_{n-2}\right) \in \rho$, then $b_{2} \in R_{1}\left(x_{n+1}\right)=L\left(x_{n+1}\right)$, so $x_{n+1} \in R\left(b_{2}\right)=$ $L\left(b_{2}\right) . a \in L\left(x_{n+1}\right)$ and $x_{n+1} \in L\left(b_{2}\right)$, by Lemma 2.7, $a \in L\left(b_{2}\right)$. $a \in$ $L\left(b_{2}\right)$ and $b_{2} \in L\left(x_{n+1}\right)$ from $(*)$ implies $a \in f_{\rho}\left(x_{1}, f_{\rho}\left(x_{2}, \ldots, x_{n+1}\right)\right.$, $\left.x_{n+2}, \ldots, x_{2 n-1}\right)$.

$\left(i_{n+2}\right)$ If $a \in L\left(x_{n+2}\right)$, then by $(*)$, we have $a \in f_{\rho}\left(x_{1}, f_{\rho}\left(x_{2}, \ldots, x_{n+1}\right), x_{n+2}\right.$, $\left.\ldots, x_{2 n-1}\right)$.

$\left(i_{2 n-1}\right)$ If $a \in L\left(x_{2 n-1}\right)$, then by $(*)$ we have $a \in f_{\rho}\left(x_{1}, f_{\rho}\left(x_{2}, \ldots, x_{n+1}\right)\right.$, $\left.x_{n+2}, \ldots, x_{2 n-1}\right)$.

The proofs of the other inclusions are similar and with long computations. It remains to check the condition of the join $n$-ary space. Set $a, b_{1}, \ldots, b_{n-1}, c, d_{1}$, $\ldots, d_{n-1} \in H$ such that $a / b_{1}^{n-1} \cap c / d_{1}^{n-1} \neq \emptyset$. Then, there exists $x \in a / b_{1}^{n-1} \cap$ $c / d_{1}^{n-1}$. Hence,

$$
\begin{aligned}
& x \in a / b_{1}^{n-1} \Rightarrow a \in f_{\rho}\left(x, b_{1}, \ldots, b_{n-1}\right)=L(x) \cup L\left(b_{1}\right) \cup \cdots \cup L\left(b_{n-1}\right), \\
& x \in c / d_{1}^{n-1} \Rightarrow c \in f_{\rho}\left(x, d_{1}, \ldots, d_{n-1}\right)=L(x) \cup L\left(d_{1}\right) \cup \cdots \cup L\left(d_{n-1}\right) .
\end{aligned}
$$

Now, we consider the following situations:

$$
\begin{array}{rl}
1 & a \in L(x), c \in L(x) \Rightarrow x \in L(a), x \in L(c) \Rightarrow x \in\left[L(a) \cup L\left(d_{1}\right) \cup\right. \\
& \left.\ldots \cup L\left(d_{n-1}\right)\right] \cap\left[L\left(b_{1}\right) \cup \cdots \cup L\left(b_{n-1}\right) \cup L(c)\right]=f_{\rho}\left(a, d_{1}, \ldots, d_{n-1}\right) \cap \\
& f_{\rho}\left(b_{1}, \ldots, b_{n-1}, c\right) .
\end{array}
$$

2 If $a \in L(x)$ and $c \in L\left(d_{i}\right), i \in\{1, \ldots, n-1\}$. Since $c \in L(c)$ (by reflexivity), it follows that

$$
c \in\left[L(a) \cup L\left(d_{1}\right) \cup \ldots \cup L\left(d_{n-1}\right)\right] \cap\left[L\left(b_{1}\right) \cup \ldots \cup L\left(b_{n-1}\right) \cup L(c)\right] .
$$

3 If $a \in L\left(b_{i}\right)$ and $c \in L(x), i \in\{1, \ldots, n-1\}$, then $b_{i} \in R(a)=L(a)$. Since $b_{i} \in L\left(b_{i}\right)$ (by reflexivity), it follows that

$b_{i} \in\left[L(a) \cup L\left(d_{1}\right) \cup \ldots \cup L\left(d_{n-1}\right)\right] \cap\left[L\left(b_{1}\right) \cup \ldots \cup L\left(b_{n-1}\right) \cup L(c)\right]$.

4 If $a \in L\left(b_{i}\right)$ and $c \in L\left(d_{j}\right), i, j \in\{1, \ldots, n-1\}$, then $b_{i} \in R(a)=L(a)$, since $b_{i} \in L\left(b_{i}\right)$ (by reflexivity), it follows that

$$
b_{i} \in\left[L(a) \cup L\left(d_{1}\right) \cup \ldots \cup L\left(d_{n-1}\right)\right] \cap\left[L\left(b_{1}\right) \cup \ldots \cup L\left(b_{n-1}\right) \cup L(c)\right] .
$$

Since for $a, b_{1}, \ldots, b_{n-1}, c, d_{1}, \ldots, d_{n-1} \in H$ such that $a / b_{1}^{n-1} \cap c / d_{1}^{n-1} \neq \emptyset$, we have $f_{\rho}\left(a, d_{1}, \ldots, d_{n-1}\right) \cap f_{\rho}\left(b_{1}, \ldots, b_{n-1}, c\right) \neq \emptyset$, so $\left(H, f_{\rho}\right)$ is a join $n$-ary space.

Remark 2. Let $\rho$ be an $n$-ary reflexive relation on a set $H$. By Lemma 2.7, if $\rho$ is $n$-transitive, then $\rho$ satisfies the following property:

$(\mathbf{T})$

for any $a, x, u \in H$ and $i \in\{1, \ldots, n-1\}$ such that $a \in L_{i}(u)\left[a \in R_{i}(u)\right]$ and $u \in L_{i}(x)\left[u \in R_{i}(x)\right]$, it follows that $a \in L_{i}(x)\left[a \in R_{i}(x)\right]$. 
Theorem 2.10. Let $\rho$ be an $n$-ary relation on $H$ such that $x \in L_{i}(x)=R_{j}(x)$ for any $x \in H$ and $1 \leq i, j \leq m$. If $\rho$ satisfies the properties $(\mathbf{T})$, then $f_{\rho}$ is associative and so $\left(H, f_{\rho}\right)$ is a join n-ary spaces.

Proof. The reproducibility follows from Theorem 2.4. Suppose that $f_{\rho}$ is not associative. Then, there exists $x_{1}^{2 n-1}$ such that $f_{\rho}\left(f_{\rho}\left(x_{1}, \ldots, x_{n}\right), x_{n+1}, \ldots\right.$, $\left.x_{2 n-1}\right)$ is not equal to

$$
\begin{aligned}
& f_{\rho}\left(x_{1}, f_{\rho}\left(x_{2}, \ldots, x_{n+1}\right), x_{n+2}, \ldots, x_{2 n-1}\right) \text { or } \\
& f_{\rho}\left(x_{1}, x_{2}, f_{\rho}\left(x_{3}, \ldots, x_{n+2}\right), x_{n+3}, \ldots, x_{2 n-1}\right) \ldots \text { or } \\
& f_{\rho}\left(x_{1}, \ldots, x_{n-1}, f_{\rho}\left(x_{n}, \ldots, x_{2 n-1}\right)\right) .
\end{aligned}
$$

Suppose that there exists $u \in f_{\rho}\left(f_{\rho}\left(x_{1}, \ldots, x_{n}\right), x_{n+1}, \ldots, x_{2 n-1}\right)$ such that

$$
u \notin f_{\rho}\left(x_{1}, f_{\rho}\left(x_{2}, \ldots, x_{n+1}\right), x_{n+2}, \ldots, x_{2 n-1}\right)
$$

or vice versa. We consider the first situation: it follows that there exists $t \in f_{\rho}\left(x_{1}, \ldots, x_{n}\right)$ such that $u \in L(t) \cup L\left(x_{n+1}\right) \cup \cdots \cup L\left(x_{2 n-1}\right)$ and for any $s \in f_{\rho}\left(x_{2}, \ldots, x_{n+1}\right), u \notin L\left(x_{1}\right) \cup L(s) \cup L\left(x_{n+2}\right) \cup \cdots \cup L\left(x_{2 n-1}\right)$. Now, we distinguish the following situation:

(1) If $u \in L(t)$ and $t \in L\left(x_{1}\right)$, then $u \in L\left(x_{1}\right)$, so $u \in L\left(x_{1}\right) \cup \cdots \cup L\left(x_{2 n-1}\right)$.

(2) If $u \in L(t)$ and $t \in L\left(x_{2}\right)$, then $u \in L\left(x_{2}\right)$. Since $x_{2} \in f_{\rho}\left(x_{2}, \ldots, x_{n+1}\right)$. Thus, $u \in L\left(x_{1}\right) \cup L\left(x_{2}\right) \cup L\left(x_{n+2}\right) \cup \cdots \cup L\left(x_{2 n-1}\right)$.

(n) If $u \in L(t)$ and $t \in L\left(x_{n}\right)$, then $u \in L\left(x_{n}\right)$. Since $x_{n} \in f_{\rho}\left(x_{2}, \ldots, x_{n+1}\right)$, $u \in L\left(x_{1}\right) \cup L\left(x_{n}\right) \cup L\left(x_{n+2}\right) \cup \cdots \cup L\left(x_{2 n-1}\right)$.

$(n+1)$ If $u \in L\left(x_{n+1}\right)$, then $u \in L\left(x_{1}\right) \cup L\left(x_{n+1}\right) \cup \cdots \cup L\left(x_{2 n-1}\right)$, since $x_{n+1} \in f_{\rho}\left(x_{2}, \ldots, x_{n+1}\right)$,

$(n+2)$ If $u \in L\left(x_{n+2}\right)$, then $u \in L\left(x_{1}\right) \cup L(s) \cup L\left(x_{n+2}\right) \cup \cdots \cup L\left(x_{2 n-1}\right)$.

$(2 n-1)$ If $u \in L\left(x_{2 n-1}\right)$, then $u \in L\left(x_{1}\right) \cup L(s) \cup L\left(x_{n+2}\right) \cup \cdots \cup L\left(x_{2 n-1}\right)$.

For the all cases, we obtain a contradiction with the fact

$$
u \notin L\left(x_{1}\right) \cup L(s) \cup L\left(x_{n+2}\right) \cup \cdots \cup L\left(x_{2 n-1}\right)
$$

for any $s \in f_{\rho}\left(x_{2}, \ldots, x_{n+1}\right)$. The proofs of the other inclusions are similar. Therefore, $f_{\rho}$ is associative.

Example 8. Let $H=\{0,1,2\}$ and

$$
\rho=\{(\underbrace{0, \ldots, 0}_{n}),(1,2, \ldots, 1,2),(2,1, \ldots, 2,1),(\underbrace{1, \ldots, 1}_{n-1}, 2),(\underbrace{2, \ldots, 2}_{n-1}, 1) .
$$

Then, we have

\begin{tabular}{|c|c|c|c|c|c|c|c|c|c|}
\hline & $L_{1}$ & $L_{2}$ & $L_{3}$ & $\ldots$ & $L_{m}$ & $R_{1}$ & $R_{2}$ & $\ldots$ & $R_{m}$ \\
\hline 0 & $\{0\}$ & $\{0\}$ & $\{0\}$ & $\ldots$ & $\{0\}$ & $\{0\}$ & $\{0\}$ & $\ldots$ & $\{0\}$ \\
\hline 1 & $\{1,2\}$ & $\{1,2\}$ & $\{1,2\}$ & $\ldots$ & $\{1,2\}$ & $\{1,2\}$ & $\{1,2\}$ & $\ldots$ & $\{1,2\}$ \\
\hline 2 & $\{1,2\}$ & $\{1,2\}$ & $\{1,2\}$ & $\ldots$ & $\{1,2\}$ & $\{1,2\}$ & $\{1,2\}$ & $\ldots$ & $\{1,2\}$ \\
\hline
\end{tabular}


for every $x \in H$ and $1 \leq i, j \leq m$, we have $L_{i}(x)=R_{j}(x)$. So,

$$
f_{\rho}\left(x_{1}, \ldots, x_{n}\right)= \begin{cases}\{0\} & \text { if }\left\{x_{1}, \ldots, x_{n}\right\}=\{0\}, \\ \{1,2\} & \text { if }\left\{x_{1}, \ldots, x_{n}\right\} \subseteq\{1,2\}, \\ \{0,1,2\} & \text { otherwise. }\end{cases}
$$

It is not difficult to see that $\left(H, f_{\rho}\right)$ is a join $n$-ary space.

\section{3. $n$-ary $\boldsymbol{H}_{\boldsymbol{v}}$-groups associated with $\boldsymbol{n}$-ary relations}

Given an $n$-ary hypergroupoid $(H, f)$, we may consider the $(n+1)$-ary relation $\rho_{k}$ on $H$ associated with the $n$-ary hyperoperation $f$ as follows

$$
\left(x_{1}, \ldots, x_{n+1}\right) \in \rho_{k} \Leftrightarrow x_{k} \in f\left(x_{1}^{k-1}, x_{k+1}^{n+1}\right) .
$$

This is the most natural way to define an $(n+1)$-ary relation associated with an $n$-ary hyperoperation. If $(H, f)$ is an $n$-ary hypergroup, then $\rho_{k}$ satisfies the following conditions:

(1) For all $x_{1}, \ldots, x_{n} \in H$, there exists at least one element $x \in H$ such that $\left(x_{1}^{k-1}, x, x_{k}^{n}\right) \in \rho_{k}$.

(2) If, for $x_{1}, \ldots, x_{2 n+1}, z \in H$, there exists $x \in H$ such that for any $k \leq i$ and $k \leq j$, we have $\left(x_{1}^{k-1}, z, x_{k}^{i-1}, x, x_{n+i}^{2 n-1}\right) \in \rho_{k}$ and $\left(x_{i}^{i+k-2}, x, x_{i+k-1}^{i+n-1}\right)$ $\in \rho_{k}$, then there exists $y \in H$ such that $\left(x_{1}^{k-1}, z, x_{k}^{j-1}, y, x_{n+j}^{2 n-1}\right) \in \rho_{k}$ and $\left(x_{j}^{j+k-2}, x, x_{j+k-1}^{j+n-1}\right) \in \rho_{k}$, and conversely.

(3) If, for $x_{1}, \ldots, x_{2 n+1}, z \in H$, there exists $x \in H$ such that for any $k \leq i$ and $k>j$, we have $\left(x_{1}^{k-1}, z, x_{k}^{i-1}, x, x_{n+i}^{2 n-1}\right) \in \rho_{k}$ and $\left(x_{i}^{i+k-2}, x, x_{i+k-1}^{i+n-1}\right)$ $\in \rho_{k}$, then there exists $y \in H$ such that $\left(x_{1}^{j-1}, y, x_{n+j}^{n+k-2}, z, x_{n+k-1}^{2 n-1}\right) \in$ $\rho_{k}$ and $\left(x_{j}^{j+k-2}, x, x_{j+k-1}^{j+n-1}\right) \in \rho_{k}$, and conversely.

(4) If, for $x_{1}, \ldots, x_{2 n+1}, z \in H$, there exists $x \in H$ such that for any $k>i$ and $k>j$, we have $\left(x_{1}^{i-1}, y, x_{n+i}^{n+k-2}, z, x_{n+k-1}^{2 n-1}\right) \in \rho_{k}$ and $\left(x_{i}^{i+k-2}, x, x_{i+k-1}^{i+n-1}\right) \in \rho_{k}$, then there exists $y \in H$ such that $\left(x_{1}^{j-1}, y\right.$, $\left.x_{n+j}^{n+k-2}, z, x_{n+k-1}^{2 n-1}\right) \in \rho_{k}$ and $\left(x_{j}^{j+k-2}, x, x_{j+k-1}^{j+n-1}\right) \in \rho_{k}$, and conversely.

(5) For all $x_{1}^{n} \in H$ and $1 \leq i \leq n$, there exists $x \in H$ such that $\left(x_{1}^{i-1}, x, x_{i}^{n}\right)$ $\in \rho_{k}$.

Conversely, if $\rho$ is an $(n+1)$-ary relation on a set $H$ such that the conditions (1)-(5) are satisfied, then we take the $n$-ary hyperoperation

$$
f_{k}\left(x_{1}, \ldots, x_{n}\right)=\left\{z \in H \mid\left(x_{1}^{k-1}, z, x_{k}^{n}\right) \in \rho\right\} .
$$

Hence, $\left(H, f_{k}\right)$ is an $n$-ary hypergroup. Let $\sigma_{n}$ be an $n$-ary relation on a set $H$. We associate an $(n+1)$-ary relation denoted by $\sigma_{n+1} \subseteq H^{n+1}$ as follows:

$$
\left(x_{1}, \ldots, x_{n+1}\right) \in \sigma_{n+1} \Longleftrightarrow \forall 1 \leq i \leq n+1,\left(x_{1}^{i-1}, x_{i+1}^{n+1}\right) \in \sigma_{n} .
$$

Proposition 3.1. The unique $(n+1)$-ary relation $\sigma_{n+1}$ obtained from an $n$-ary relation $\sigma_{n}$ using the method (1) and such that

$$
\left(x_{1}^{i-1}, x_{i+1}^{n+1}\right) \in H^{n}, \quad \exists x_{i} \in H:\left(x_{1}, \ldots, x_{n+1}\right) \in \sigma_{n+1}
$$


is the total relation $\sigma_{n+1}=\underbrace{H \times \cdots \times H}_{n+1}$.

Proof. The condition (2) is equivalent to the following one: for any $\left(x_{1}, \ldots, x_{n}\right)$ $\in H^{n},\left(x_{1}, \ldots, x_{n}\right) \in \sigma_{n}$, so the $n$-ary relation $\sigma_{n}$ is the total relation $\underbrace{H \times \cdots \times H}_{n}$.

Thus, for any $\left(x_{1}, \ldots, x_{n+1}\right) \in H^{n+1}$ and for any $1 \leq i \leq n+1$, we have $\left(x_{1}^{i-1}, x_{i+1}^{n+1}\right) \in H^{n}=\sigma_{n}$. Therefore, by using the method $(1),\left(x_{1}, \ldots, x_{n+1}\right) \in$ $\sigma_{n+1}$. So, $H^{n+1} \subseteq \sigma_{n+1}$. Therefore, $\sigma_{n+1}=H^{n+1}$.

Moreover, the $n$-ary hypergroupoid obtained from $\sigma_{n+1}$ taking

$$
f\left(x_{1}^{i-1}, x_{i+1}^{n+1}\right)=\left\{x_{i} \in H \mid\left(x_{1}, \ldots, x_{n+1}\right) \in \sigma_{n+1}\right\}
$$

is the total $n$-ary hypergroup on $H$. Conversely, with any $(n+1)$-ary relation $\rho_{n+1}$ on $H$, we associate an $n$-ary relation $\rho_{n} \subseteq H^{n}$ as follows:

$$
\left(x_{1}^{i-1}, x_{i+1}^{n+1}\right) \in \rho_{n} \Longleftrightarrow \exists x_{i} \in H:\left(x_{1}, \ldots, x_{n+1}\right) \in \rho_{n+1} .
$$

Let $(H, f)$ be an arbitrary $n$-ary hypergroupoid which determines the $(n+1)$ ary relation $\rho_{n+1}$ defined by

$$
\left(x_{1}, \ldots, x_{n+1}\right) \in \rho_{n+1} \Leftrightarrow x_{i} \in f\left(x_{1}^{i-1}, x_{i+1}^{n+1}\right) \text { for some } 1 \leq i \leq n .
$$

Proposition 3.2. The unique $n$-ary relation $\rho_{n}$ obtained from an $(n+1)$-ary relation $\rho_{n+1}$ using the method (3) and such that

$$
\left(x_{1}, \ldots, x_{n+1}\right) \in \rho_{n+1} \Leftrightarrow x_{i} \in f\left(x_{1}^{i-1}, x_{i+1}^{n+1}\right),
$$

is the total relation $\rho_{n}=\underbrace{H \times \cdots \times H}_{n}$.

Proof. Since $(H, f)$ is an $n$-ary hypergroupoid, it follows that, for any $\left(x_{1}^{i-1}\right.$, $\left.x_{i+1}^{n+1}\right) \in H^{n}$, there exists $x_{i} \in H$ such that $x_{i} \in f\left(x_{1}^{i-1}, x_{i+1}^{n+1}\right)$, that is $\left(x_{1}, \ldots, x_{n+1}\right) \in \rho_{n+1}$. Therefore, for any $\left(x_{1}^{i-1}, x_{i+1}^{n+1}\right) \in H^{n}$, we obtain $\left(x_{1}^{i-1}, x_{i+1}^{n+1}\right) \in \rho_{n}$, that is $\rho_{n}=\underbrace{H \times \cdots \times H}_{n}$.

Definition 3.3. Let $(H, f)$ be an $n$-ary hypergroup, such that the $n$-ary hyperoperation $f$ is constructed by the $(n+1)$-ary relation $\rho$, which satisfy the conditions (1)-(3). We define an $(n+1)$-ary hyperoperation:

$$
h\left(x_{1}, \ldots, x_{n+1}\right)=\bigcup_{i=1}^{n+1} f\left(x_{1}^{i-1}, x_{i+1}^{n+1}\right) \text { for all } x_{1}, \ldots, x_{n+1} \in H .
$$

Theorem 3.4. Let $h$ be the $(n+1)$-ary hyperoperation in Definition 3.3. Then, $(H, h)$ is an $(n+1)$-ary $H_{v}$-group.

Proof. Since $(H, f)$ is an $n$-ary hypergroup, $(H, h)$ is an $(n+1)$-ary hypergroupoid. Let $x_{1}, \ldots, x_{n+1} \in H$. Then, producibility of $(H, f)$ implies that

$\left(i_{1}\right)$

$$
h\left(H, x_{2}, \ldots, x_{n+1}\right)
$$




$$
=f\left(x_{2}, \ldots, x_{n+1}\right) \cup f\left(H, x_{3}, \ldots, x_{n+1}\right) \cup \ldots \cup f\left(H, x_{2}, \ldots, x_{n}\right)=H,
$$

$\left(i_{2}\right)$

$$
h\left(x_{1}, H, x_{3}, \ldots, x_{n+1}\right)
$$$$
=f\left(H, x_{3}, \ldots, x_{n+1}\right) \cup f\left(x_{1}, x_{3}, \ldots, x_{n+1}\right) \cup \ldots \cup f\left(x_{1}, H, \ldots, x_{n}\right)=H,
$$

$\left(i_{n+1}\right)$

$$
\begin{aligned}
& h\left(x_{1}, x_{2}, \ldots, x_{n}, H\right) \\
= & f\left(x_{2}, x_{3}, \ldots, x_{n}, H\right) \cup f\left(x_{1}, x_{3}, \ldots, x_{n}, H\right) \cup \ldots \cup f\left(x_{1}, \ldots, x_{n}\right)=H .
\end{aligned}
$$

Thus, $(H, h)$ is productive. Now, we prove that $h$ is weakly associative. Suppose that $x_{1}^{2 n+1} \in H$. Then,

$\left(i_{1}\right)$

$$
\begin{aligned}
h\left(h\left(x_{1}^{n+1}\right), x_{n+2}, \ldots, x_{2 n+1}\right) & =h\left(\bigcup_{i=1}^{n+1} f\left(x_{1}^{i-1}, x_{i+1}^{n+1}\right), x_{n+2}, \ldots, x_{2 n+1}\right) \\
& =h\left(g, x_{n+2}, \ldots, x_{2 n+1}\right) \\
& \supseteq f\left(g, x_{n+3}, \ldots, x_{2 n+1}\right) \\
& \supseteq f\left(f\left(x_{2}, \ldots, x_{n+1}\right), x_{n+3}, \ldots, x_{2 n+1}\right),
\end{aligned}
$$

$\left(i_{2}\right)$

$$
\begin{aligned}
& h\left(x_{1}, h\left(x_{2}^{n+2}\right), x_{n+3}, \ldots, x_{2 n+1}\right)=h\left(x_{1}, \bigcup_{i=1}^{n+1} f\left(x_{2}^{i}, x_{i+2}^{n+2}\right), x_{n+3}, \ldots, x_{2 n+1}\right) \\
&=h\left(x_{1}, g, x_{n+3}, \ldots, x_{2 n+1}\right) \\
& \supseteq f\left(g, x_{n+3}, \ldots, x_{2 n+1}\right) \\
& \supseteq f\left(f\left(x_{2}, \ldots, x_{n+1}\right), x_{n+3}, \ldots, x_{2 n+1}\right), \\
& \vdots \\
&\left(i_{n+1}\right) \quad \\
& h\left(x_{1}, \ldots x_{n}, h\left(x_{n+1}, \ldots, x_{2 n+1}\right)\right)=h\left(x_{1}, \ldots x_{n}, \bigcup_{i=1}^{n+1} f\left(x_{n+1}^{n+i-1}, x_{n+i+1}^{2 n+1}\right)\right) \\
&=h\left(x_{1}, \ldots x_{n}, g\right) \\
& \supseteq f\left(x_{2}, \ldots, x_{n}, g\right) \\
& \supseteq f\left(x_{2}, \ldots, x_{n}, f\left(x_{n+1}, x_{n+3}, \ldots, x_{2 n+1}\right)\right) .
\end{aligned}
$$

Therefore,

$$
\bigcap_{i=1}^{2 n+1} h\left(x_{1}^{i-1}, h\left(x_{i}^{n+i-1}\right), x_{n+i}^{2 n+1}\right) \neq \emptyset .
$$


This implies that $(n+1)$-ary hypergroupoid $(H, h)$ is weakly associative.

Example 9. Let $H=\left\{a_{1}, \ldots, a_{n}\right\}$ and $f$ be an $n$-ary relation on $H$ such that

$$
\begin{aligned}
& f(\underbrace{a_{1}, \ldots, a_{1}}_{n})=\left\{a_{n}\right\}, f(\underbrace{a_{2}, \ldots, a_{2}}_{n})=\left\{a_{n-1}\right\} \\
& \vdots \\
& f(\underbrace{a_{n-1}, \ldots, a_{n-1}}_{n})=\left\{a_{2}\right\}, f(\underbrace{a_{n}, \ldots, a_{n}}_{n})=\left\{a_{1}\right\} \\
& f\left(a_{1}^{a_{1}, \ldots, a_{n}}\right)=f(\underbrace{a_{1}, \ldots, a_{1}}_{n}) \cup \cdots \cup f(\underbrace{a_{n}, \ldots, a_{n}}_{n}) .
\end{aligned}
$$

Then, $(H, f)$ is an $n$-ary hypergroupoid. Now, if

$$
h\left(a_{1}, \ldots, a_{n+1}\right)=\bigcup_{i=1}^{n+1} f\left(a_{1}^{i-1}, a_{i+1}^{n+1}\right),
$$

then the $(n+1)$-ary hypergroupoid $(H, h)$ is an $(n+1)$-ary $H_{v}$-group, but it is not an $(n+1)$-ary hypergroup. For instance,

$$
\begin{aligned}
& h(\underbrace{a_{1}, \ldots, a_{1}}_{n}, h(\underbrace{a_{1}, \ldots, a_{1}}_{n}, a_{2})) \\
= & h(\underbrace{a_{1}, \ldots, a_{1}}_{n},\left\{a_{n}, a_{n-1}\right\}) \\
= & h(\underbrace{a_{1}, \ldots, a_{1}}_{n+1}) \cup h(\underbrace{a_{n-1}, \ldots, a_{n-1}}_{n+1}) \cup h(\underbrace{a_{n}, \ldots, a_{n}}_{n+1})=\left\{a_{1}, a_{2}, a_{n}\right\}, \\
& h(\underbrace{a_{1}, \ldots, a_{1}}_{n-1}, h(\underbrace{a_{1}, \ldots, a_{1}}_{n+1}), a_{2}) \\
= & h(\underbrace{a_{1}, \ldots, a_{1}}_{n-1},\left\{a_{n}\right\}, a_{2}) \\
= & f(\underbrace{a_{1}, \ldots, a_{1}}_{n+1}) \cup f(\underbrace{a_{n}, \ldots, a_{n}}_{n+1}) \cup f(\underbrace{a_{2}, \ldots, a_{2}}_{n+1})=\left\{a_{1}, a_{n-1}, a_{n}\right\} .
\end{aligned}
$$

Let $\rho$ be a binary relation on a non-empty set $H$. For any $a \in H$, we denote $f_{\rho}(\underbrace{a, \ldots, a}_{n})=\{y \mid(a, y) \in \rho\}$, and for any $a_{1}, \ldots, a_{n} \in H$,

$$
f_{\rho}\left(a_{1}, a_{2}, \ldots, a_{n}\right)=f_{\rho}(\underbrace{a_{1}, \ldots, a_{1}}_{n}) \cup f_{\rho}(\underbrace{a_{2}, \ldots, a_{2}}_{n}) \cup \cdots \cup f_{\rho}(\underbrace{a_{n}, \ldots, a_{n}}_{n}) .
$$

Definition 3.5. Let $\rho$ be a binary relation on a set $H$. We define the $(n+1)$-ary hyperoperation $\mathbb{F}_{\rho}$ as follows:

$$
\mathbb{F}_{\rho}\left(x_{1}, \ldots, x_{n+1}\right)=\bigcup_{i=1}^{n+1} f_{\rho}(\underbrace{x_{i}, \ldots, x_{i}}_{n})=\bigcup_{i=1}^{n+1} U_{x_{i}}
$$


when $U_{x_{i}}=f_{\rho}(\underbrace{x_{i}, \ldots, x_{i}}_{n})$.

Theorem 3.6. Let $\rho$ be a binary relation on a set $H$, with full domain and full range. Let $\mathbb{F}_{\rho}$ be the $(n+1)$-ary hyperoperation in Definition 3.5. Then, $\left(H, \mathbb{F}_{\rho}\right)$ is an $(n+1)$-ary $H_{v}$-group.

Proof. Since $D(\rho)=H$ and $\left(H, f_{\rho}\right)$ is an $n$-ary hypergroupoid, $\left(H, \mathbb{F}_{\rho}\right)$ is an $(n+1)$-ary hypergroupoid. Let $x_{1}, \ldots, x_{n} \in H$. Then,

$$
\begin{aligned}
U_{H}=f_{\rho}(\underbrace{H, \ldots, H}_{n}) & =\{y \in H \mid(H, y) \in \rho\}=\{y \in H \mid \exists x \in H,(x, y) \in \rho\} \\
& =D(\rho)=H .
\end{aligned}
$$

So, for $x_{1}, \ldots, x_{n} \in H$ we have

$$
\mathbb{F}_{\rho}\left(H, x_{2}, \ldots, x_{n+1}\right)=U_{H} \cup \bigcup_{i=2}^{n+1} U_{x_{i}}=H .
$$

By the similar way, we have $\mathbb{F}_{\rho}\left(x_{1}, H, x_{3}, \ldots, x_{n+1}\right)=\cdots=\mathbb{F}_{\rho}\left(x_{1}, \ldots, x_{n}, H\right)$ $=H$. Now, we prove that $\mathbb{F}_{\rho}$ is weakly associative. If $x_{1}^{2 n+1} \in H$, then,

$\left(i_{1}\right)$

$$
\begin{aligned}
\mathbb{F}_{\rho}\left(\mathbb{F}_{\rho}\left(x_{1}^{n+1}\right), x_{n+2}, \ldots, x_{2 n+1}\right) & =\mathbb{F}_{\rho}\left(U_{x_{1}} \cup \cdots \cup U_{x_{n+1}}, x_{n+2}, \ldots, x_{2 n+1}\right) \\
& =\bigcup_{g \in U_{x_{1}} \cup \cdots \cup U_{x_{n+1}}} \mathbb{F}_{\rho}\left(g, x_{n+2}, \ldots, x_{2 n+1}\right) \\
& \supseteq \bigcup_{g \in U_{x_{n+1}}} \mathbb{F}_{\rho}\left(g, x_{n+2}, \ldots, x_{2 n+1}\right) \\
& =\mathbb{F}_{\rho}\left(U_{x_{n+1}}, x_{n+2}, \ldots, x_{2 n+1}\right) \\
& \supseteq \bigcup_{g \in U_{x_{n+1}}} U_{g},
\end{aligned}
$$

$\left(i_{2}\right)$

$$
\begin{aligned}
\mathbb{F}_{\rho}\left(x_{1}, \mathbb{F}_{\rho}\left(x_{2}^{n+2}\right), x_{n+3}, \ldots, x_{2 n+1}\right) & =\mathbb{F}_{\rho}\left(x_{1}, U_{x_{2}} \cup \cdots \cup U_{x_{n+2}}, x_{n+3}, \ldots, x_{2 n+1}\right) \\
& =\bigcup_{g \in U_{x_{2}} \cup \cdots \cup U_{x_{n+2}}} \mathbb{F}_{\rho}\left(x_{1}, g, x_{n+3}, \ldots, x_{2 n+1}\right) \\
& \supseteq \bigcup_{g \in U_{x_{n+1}}} \mathbb{F}_{\rho}\left(x_{1}, g, x_{n+3}, \ldots, x_{2 n+1}\right) \\
& =\mathbb{F}_{\rho}\left(x_{1}, U_{x_{n+1}}, x_{n+3}, \ldots, x_{2 n+1}\right) \\
& \supseteq \bigcup_{g \in U_{x_{n+1}}} U_{g}
\end{aligned}
$$


$\left(i_{n+1}\right)$

$$
\begin{aligned}
\mathbb{F}_{\rho}\left(x_{1}, \ldots, x_{n}, \mathbb{F}_{\rho}\left(x_{n+1}^{2 n+1}\right)\right) & =\mathbb{F}_{\rho}\left(x_{1}, \ldots, x_{n}, U_{x_{n+1}} \cup \cdots \cup U_{x_{2 n+1}}\right) \\
& =\bigcup_{g \in U_{x_{n+1}} \cup \ldots \cup U_{x_{2 n+1}}} \mathbb{F}_{\rho}\left(x_{1}, \ldots, x_{n}, g\right) \\
& \supseteq \bigcup_{g \in U_{x_{n+1}}} \mathbb{F}_{\rho}\left(x_{1}, \ldots, x_{n}, g\right) \\
& =\mathbb{F}_{\rho}\left(x_{1}, \ldots, x_{n}, U_{x_{n+1}}\right) \\
& \supseteq \bigcup_{g \in U_{x_{n+1}}} U_{g} .
\end{aligned}
$$

It follows that $\left(H, \mathbb{F}_{\rho}\right)$ is an $(n+1)$-ary $H_{v}$-group.

Example 10. Let $H=\{1, \ldots, n\}, n \geq 4$ and $\rho=\{(1, n), \ldots,(i, n-i+$ $1), \ldots,(n, 1)\}$ be a binary relation on a set $H$, with full domain and full range. Then,

$$
\begin{aligned}
& U_{1}=f_{\rho}(\underbrace{1, \ldots, 1}_{n})=\{n\}, \quad U_{2}=f_{\rho}(\underbrace{2, \ldots, 2}_{n})=\{n-1\}, \ldots, \\
& U_{n-1}=f_{\rho}(\underbrace{n-1, \ldots, n-1}_{n})=\{2\}, U_{n}=f_{\rho}(\underbrace{n, \ldots, n}_{n})=\{1\} .
\end{aligned}
$$

The properties of $\left(H, f_{\rho}\right)$, where $f_{\rho}\left(x_{1}, \ldots, x_{n}\right)=\bigcup_{i=1}^{n} U_{x_{i}}$, as $n$-ary $H_{v}$-group, whit rarely computations guarantee that the $\left(H, \mathbb{F}_{\rho}\right)$ is an $(n+1)$-ary $H_{v}$-group properties. But $\left(H, \mathbb{F}_{\rho}\right)$ is not an $(n+1)$-ary hypergroup.

For instance

$$
\begin{aligned}
\mathbb{F}_{\rho}(\underbrace{1, \ldots, 1}_{n}, \mathbb{F}_{\rho}(\underbrace{1, \ldots, 1}_{n}, 2)) & =\mathbb{F}_{\rho}(\underbrace{1, \ldots, 1}_{n}, U_{1} \cup U_{2}) \\
& =\mathbb{F}_{\rho}(\underbrace{1, \ldots, 1}_{n},\{n, n-1\}) \\
& =U_{1} \cup U_{n-1}^{U_{n-1}} \cup U_{n}=\{1,2, n\}, \\
\mathbb{F}_{\rho}(\underbrace{1, \ldots, 1}_{n-1}, \mathbb{F}_{\rho}(\underbrace{1, \ldots, 1}_{n+1}), 2) & =\mathbb{F}_{\rho}(\underbrace{1, \ldots, 1}_{n-1}, U_{1}, 2) \\
& =\mathbb{F}_{\rho}(\underbrace{1, \ldots, 1}_{n-1},\{n\}, 2) \\
& =U_{1} \cup U_{2} \cup U_{n}=\{1, n-1, n\} .
\end{aligned}
$$

\section{References}

[1] P. Corsini, Binary relations and hypergroupoids, Ital. J. Pure Appl. Math. 7 (2000), $11-18$.

[2] P. Corsini and V. Leoreanu, Hypergroups and binary relations, Algebra Universalis $\mathbf{4 3}$ (2000), no. 4, 321-330. 
[3] I. Cristea and M. Stefanescu, Hypergroups and n-ary relations, European J. Combin. 31 (2010), no. 3, 780-789.

[4] B. Davvaz, W. A. Dudek, and T. Vougiouklis, A generalization of $n$-ary algebraic systems, Comm. Algebra 37 (2009), no. 4, 1248-1263.

[5] B. Davvaz and V. Leoreanu-Fotea, Binary relations for ternary semihypergroups, Comm. Algebra 38 (2010), no. 10, 3621-3636.

[6] B. Davvaz and T. Vougiouklis, n-ary hypergroups, Iran. J. Sci. Technol. Trans. A Sci. 30 (2006), no. 2, 165-174.

[7] M. De Salvo and J. Lo Faro, A new class of hypergroupoids associated to binary relations, J. Mult.-Valued Logic Soft Comput. 9 (2003), no. 4, 361-375.

[8] - Hypergroups and binary relations, Mult.-Valued Log. 8 (2002), no. 5-6, 645-657.

[9] D. Freni, A new characterization of the derived hypergroup via strongly regular equivalences, Comm. Algebra 30 (2002), no. 8, 3977-3989.

[10] V. Leoreanu-Fotea and B. Davvaz, $n$-ary hypergroups and binary relations, European J. Combin. 29 (2008), no. 5, 1207-1218.

[11] _ Join n-spaces and lattices, J. Mult.-Valued Logic Soft Comput. 15 (2009), no. 5-6, 421-432.

[12] _ Roughness in n-ary hypergroups, Information Sciences 178 (2008), no. 21, 41144124.

[13] V. Leoreanu and L. Leoreanu, Hyperstructures and binary relations, Sci. Ann. Univ. Agric. Sci. Vet. Med. 45 (2002), 69-72.

[14] F. Marty, Sur une generalization de la notion de group, 8th Congress Math Scandenaves, Stockholm, (1934), 45-49.

[15] S. Rasouli and B. Davvaz, Homomorphisms, ideals and binary relations on hyper-MV algebras, J. Mult.-Valued Logic Soft Comput. 17 (2011), no. 1, 47-68.

[16] I. G. Rosenberg, Hypergroups and Join spaces determined by relations, Ital. J. Pure Appl. Math. 4 (1998), 93-101.

[17] S. Spartalis, Hypergroupoids obtained from groupoids with binary relations, Ital. J. Pure Appl. Math. 16 (2004), 201-210.

[18] S. Spartalis and C. Mamaloukas, Hyperstructures associated with binary relations, Comput. Math. Appl. 51 (2006), no. 1, 41-50.

[19] T. Vougiouklis, Fundamental relations in hyperstructures, Bull. Greek Math. Soc. 42 (1999), 113-118.

[20] _ Hyperstructures and Their Representations, Hadronic Press Inc., Florida, 1994.

[21] A new class of hyperstructures, J. Combin. Inform. System Sci. 20 (1995), no. $1-4,229-235$.

Seid Mohammad Anvariyeh

DePARTMENT OF MATHEMATiCs

YAZD UNIVERSITY

YAZD, 89195-741, IRAN

E-mail address: anvariyeh@yazduni.ac.ir

SOMAYYeH MOMENi

Department of MAThematics

YAZD UNIVERSITY

YAZD, 89195-741, IRAN

E-mail address: smomeni47@gmail.com 